\title{
Are Older Adults' Difficulties on Emotion Recognition and Theory of Mind Tasks Due to a Decline in General Cognitive Ability?
}

Qiuyi Kong ( $\sim$ qiuyi.kong@postgrad.otago.ac.nz)

University of Otago

Nicholas Currie

University of Otago

Kangning Du

University of Otago

Ted Ruffman

University of Otago

\section{Research Article}

Keywords: General Cognition, Aging, Emotion recognition, Theory of Mind

Posted Date: January 5th, 2022

DOI: https://doi.org/10.21203/rs.3.rs-1203490/v1

License: (c) (i) This work is licensed under a Creative Commons Attribution 4.0 International License. Read Full License 


\section{Abstract}

Older adults have both worse general cognition and worse social cognition. A frequent suggestion is that worse social cognition is due to worse general cognition. However, previous studies have often provided contradictory evidence. The current study examined this issue with a more extensive battery of tasks for both forms of cognition. We gave 47 young and 40 older adults three tasks to assess general cognition (processing speed, working memory, fluid intelligence) and three tasks to assess their social cognition (emotion and theory-of-mind). Older adults did worse on all tasks and there were correlations between general and social cognition. Although working memory and fluid intelligence were unique predictors of performance on the Emotion Photos task and the Eyes task, Age Group was a unique predictor on all three social cognitiaon tasks. Thus, there were relations between the two forms of cognition but older adults continued to do worse than young adults even after accounting for general cognition. We argue that this pattern of results is due to some overlap in brain areas mediating general and social cognition, but also independence, and with a differential rate of decline in brain areas dedicated to general cognition versus social cognition.

\section{Background}

Aging is associated with worse emotion recognition (ER) relative to young adults ${ }^{1,2}$, and worse theory of mind (ToM) ${ }^{3}$. Collectively, we will refer to these abilities as social cognition. There have been different explanations given as to why older adults struggle on such tasks: they have a positivity bias that makes it difficult to process negative emotions, they have difficulty with still photos because these stimuli lack ecological validity, their difficulty stems from a failure to look at the eyes, their general cognitive decline makes it hard to identify emotions and mental states, their brain decline in frontal and temporal areas results in a decline in social cognition. These views have previously been critiqued ${ }^{4}$. For instance, older adults struggle with negative and positive emotions, they have difficulty with video stimuli not just still photos, and they have difficulty with emotion bodies and voices when a failure to look at the eyes isn't relevant. Thus, these explanations do not seem adequate, yet general cognitive decline and brain decline are known features of aging and cannot be ruled out so easily. Therefore, we consider the evidence for general cognitive decline below, and we return to the brain decline explanation in the Discussion.

\section{Does General Cognitive Decline Explain Worse Emotion Recognition in Older Adults?}

Aging is accompanied by decline in core cognitive abilities such as processing speed, working memory, and fluid intelligence. Fluid intelligence refers to the ability to solve novel reasoning problems with minimal input from prior learning such as formal schooling. Fluid intelligence benefits from a faster processing speed and better working memory ${ }^{5,6}$, with processing speed, working memory and fluid intelligence all declining in old age $\mathrm{e}^{7,8}$. Given these age-related declines in cognition, researchers have 
speculated that general cognitive decline might underlie older adults' worse social cognition. Indeed, there have now been several studies that have examined this question that we review below, beginning with ER in young and middle-aged adults.

\section{Young and middle-aged adults.}

In order to examine the role of general cognition in older adults, we first consider the role of general cognition for ER in young and middle-aged adults. A number of studies indicate that the fluid and general intelligence of young and middle-aged adults is related to their ER. For instance, ER in young adults correlates with general measures of cognition tapping memory and fluid intelligence ${ }^{9}$. Likewise, in a study of young and middle-aged adults, ER was related to general intelligence (verbal ability, induction, sequential reasoning, quantitative reasoning $)^{10}$.

These relations have been investigated more thoroughly in a recent meta-analysis ${ }^{11}$. Amongst young and middle-aged participants, finding there was a mean effect size of $r=.19$ between higher intelligence and better ER with similar effects on ER for crystallized IQ, fluid IQ, spatial ability, memory, and speed. They also directly compared those under 35 to those over 35 and found higher overall correlations in the older

group (e.g., crystallized IQ: $r_{<35}=.20, r_{>35}=.28$; fluid IQ: $r_{<35}=.18, r_{>35}=.25$ ). Although crystallized IQ (e.g., vocabulary) correlated with ER, crystallized IQ generally maintains or improves with age ${ }^{12}$. Thus, it does not seem plausible that crystallized IQ underlies the age-related decrement in ER in older adults. Instead, given (a) the relation between general cognition and ER in young adults, and (b) the decline in general cognition in older adults, it seems plausible that (c) general cognitive decline might account for worse ER in older adults. Below, we consider relevant research.

\section{Older adults.}

There are a number of studies that have obtained correlations between general cognition and ER, either in a sample of older adults, or over a group of young and older adults ${ }^{13-22}$. There are also some studies that have not obtained significant correlations ${ }^{23-25}$. The contradiction in findings is complicated further by the fact that there are differences in how researchers have examined correlations, sometimes grouping all participants regardless of age, and sometimes examining correlations just in older adults themselves. In the latter case, the correlations provide information about potential decline only within the older adult group itself, rather than across the entire age span. However, if one wants to know whether correlations explain differences between young and older adults' social cognition, it would make more sense to compute correlations over all participants, not just older adults.

Furthermore, in order to understand the relation between general and social cognition, it is necessary to do more than examine correlations. A key question is whether age-related difficulties are maintained after controlling for social cognition. Once again, however, research addressing this question is inconsistent. 
For instance, some studies indicate that age differences exist even after controlling for general cognition $14,17,21,24-27$. Yet, there are other studies where (a) age effects are eliminated for at least some of the emotion tasks administered when controlling for general cognition $16,20,28,29$, (b) age differences do not exist at all after controlling for general cognition ${ }^{15,30}$, or (c) age effects are substantially reduced. For instance, in one study there were "strong reductions" in effect size ${ }^{13,18,31}$.

Further compounding the difficulty in interpreting previous findings, there are important differences in the way in which researchers have indexed general cognition by using tests of speed, working memory, or fluid intelligence, as well as the tasks used to measure each of these abilities. Whereas it might be more informative to include multiple tests tapping different aspects of general cognitive abilities, 9 of 14 studies that have examined whether age effects are independent of general cognition have included a single task to measure a single cognitive ability ${ }^{13,18-21,25,28,30}$. Likewise, 9 of 14 studies have included a single task to measure social cognition ${ }^{13,15,16,18,27-31}$. These studies do not provide a comprehensive test of the relation between general and social cognition. What is needed, and what we provide in the present study, is an analysis with multiple tests that get at different aspects of both general and social cognition.

Adding to the confusion, inconsistencies have arisen even when the same task has been used to index general cognition. For instance, consider the studies that have given just a matrices task as a measure of general cognition. After controlling for matrices performance, three studies found age differences in social cognition ${ }^{19,21,28}$, one didn't ${ }^{15}$, two found age differences on some social tasks but not others ${ }^{20,28}$, and one found a substantially reduced difference ${ }^{13}$. Thus, even when (a) the same task - matrices - is used to index general cognition, (b) matrices is one of the primary ways of examining fluid intelligence ${ }^{32}$, and (c) fluid intelligence is one of the most consistent abilities to decline in old age ${ }^{7}$, results still seem inconsistent.

In sum, (a) general cognition and social cognition are multi-faceted but previous studies have usually used a single measure of each, and (b) when one such single measure (matrices) has been used, the link to social cognition is inconsistent. Therefore, a more conclusive test of the relation between general and social cognition requires the inclusion of multiple tasks to measure different cognitive abilities.

A similar argument applies to tasks examining ER because different tasks present different information processing demands. For instance, some have claimed that dynamic stimuli (e.g., videos) should offer older adults a more ecologically valid stimulus ${ }^{33}$, which should ease difficulty. Yet, others have found that, in general, dynamic stimuli place a heavier demand on information processing resources than static stimuli ${ }^{34}$. That is, a dynamic emotional stimulus places a heavier demand on processing speed, working memory and fluid intelligence compared to still photos, because of the need for quick processing and updating of information as the display changes from one expression to another. Consistent with this argument, dynamic stimuli are generally more difficult ${ }^{34}$, and a meta-analysis of emotion tasks indicates the same; older adults have more consistent difficulties across emotions when given emotion videos than when given emotion photos ${ }^{1}$. 
For these reasons, in our study we used three tasks each to measure different aspects of both general and social cognition (for a total of six tasks). No study has provided such an extensive battery of tasks (with five studies using a total of two tasks, seven studies using a total of three tasks, and two using four).

\section{Does General Cognitive Decline Explain Worse Theory of Mind in Older Adults?}

The previous section considered ER but a similar conflict in findings has arisen when researchers have examined the role of general cognition in older adults' ToM. For instance, an early study examined the relation between processing speed, executive functioning (the Wisconsin Card Sorting Task) and ToM in young and older adults ${ }^{35}$. The ToM task (Strange Stories) required participants to explain unusual behaviour, and both processing speed and executive functioning correlated with ToM, although older adults were still worse than young adults after accounting for general cognition. Subsequently, a review of this study and many others concluded that, "ToM may be observed to function independently from general cognition in aging, but further investigation is needed to confirm this point" (p.32) ${ }^{36}$.

Since that time, subsequent studies have produced conflicting findings. For instance, one study gave participants aged 17 to 95 the Strange Stories task, along with a Stroop task, a working memory task, and an empathy scale ${ }^{37}$. Performance on the cognitive measures as well as empathy correlated with performance on the ToM task, and when entered into a regression, explained all of the variance in ToM performance, with age not explaining significant variance.

Another study varied the executive function (EF) demands of a ToM task and found that the task with high EF demands led to age differences, whereas the task with low EF demands produced no age differences ${ }^{38}$. However, they measured ToM with 20 false and 20 true belief tasks, and this is a likely shortcoming of this study in that these tasks are passed by 4- to 5-year-old children typically, and therefore, do not provide a sensitive measure of ToM. It is probable that failure occurs only because of waning attention over 40 trials rather than a lack of conceptual insight, and this likely explains the relation to EF in this study, whereas a more conceptually demanding ToM task might be unrelated to EF.

Other researchers have examined EF abilities on tasks such as Trail Making (tapping speed and fluid IQ), Backwards Digit Span (working memory), the Tower of London (planning, inhibition), and matrices (fluid IQ) in a group of young adults ${ }^{39}$. None of these tasks correlated with performance on the Eyes task (judging complex emotions and mental states in the eyes). On the Strange stories task, only matrices correlated, but there was no older group to determine whether age differences still existed.

Still another study examined the Eyes task, along with aspects of executive function in a group of older adults $^{40}$. Block Design and inhibitory ability correlated with ToM, but there was no younger group to determine whether there were age differences having accounted for general cognition. 
In sum, research presents a rather confusing picture. Sometimes general cognition correlates with ToM and sometimes it doesn't. If general cognition correlates, sometimes age differences persist when controlling for general cognition, and sometimes they don't.

\section{Present Study}

Previous studies of the relation between general cognition and ER have usually used one task that measures a single cognitive ability, with studies having obtained inconsistent findings. Inconsistency is also the norm when one narrows the lens to look only at studies employing a single cognitive task such as matrices. Yet there are multiple cognitive skills that are relevant to social cognition, with different social cognition tasks having different cognitive demands. In an effort to provide a more comprehensive index of cognitive ability, we used three tasks, all of which measured a different cognitive skill, and with all skills thought to be central to cognitive decline: speed of processing, working memory, and fluid IQ ${ }^{6}$.

Further, in a deliberate effort to vary the cognitive demands of the tasks tapping social cognition, we also included three social tasks: the Eyes task, an Emotion Photos task, and an Emotion Morph task. The Eyes task is typically thought of as a ToM measure, although it has more recently been argued that it is better construed as a test of complex emotions ${ }^{41}$. Analysis of the 36 items indicates that 9 unambiguously measure emotion (e.g., despondent), 13 have an emotional valence (e.g., insisting), and 14 are more straightforward cognitive items (e.g., preoccupied). It might, therefore, represent something of a cross between a measure of more complex emotions and mental states, but can be construed as a more demanding task than classic ER tasks, which examine only basic emotions (anger, sadness, fear, disgust, surprise, and happiness). The Emotion Photos task is a standard ER task in which we presented still photos of the face expressing basic emotions. The Emotion Morph task presented dynamic stimuli, with emotion faces that gradually morphed from one basic emotion into a different basic emotion, and the participant's task was to identify the new emotion as quickly as possible.

These three tasks tapping social cognition had intentionally differing characteristics that allowed us to carefully examine the relation between general cognition and social cognition. Again, the Eyes task required an understanding of complex emotions and mental states, but used still photos. The Emotion Photos tasks examined basic emotions and presented still photos. Neither of these tasks had a time limit on responding or required integration of information over time. In contrast, the Emotion Morph task required both of these things. As stated above, the Emotion Morph task should place a heavier demand on processing speed, working memory and fluid intelligence because of the need for quick processing and updating of information as the display changes from one thing to another ${ }^{34}$. This is particularly so because the task was speeded, in that participants were instructed to identify the new emotion as quickly as possible. Thus, to take into account both speed and accuracy, we computed a "balanced integration score" that gave equal emphasis to each. Given that speed was integral to optimal performance, and speed is a core cognitive ability that declines with age, logic would have it that the Emotion Morph task should be more heavily correlated with general cognition than the Eyes or Emotion Photos tasks. 


\section{Results}

\section{Analysis Strategy}

We first compared young and older adults on the different tasks, then we examined correlations between age and task performance, and finally, used regression to examine whether age differences in task performance were present after controlling for performance on the cognitive measures.

\section{Age Differences in Task Performance}

Table 1 includes the descriptive statistics for the main tasks. The Balanced Integration Score for the Emotion Morph task places equal emphasis on speed and accuracy ${ }^{42}$. On all tasks, young adults performed at a higher level than older adults as measured by $t$-test.

\section{Table 1}

Descriptive Statistics

\begin{tabular}{lllll} 
& \multicolumn{2}{l}{ Young Adults } & \multicolumn{2}{l}{ Older Adults } \\
\hline Matrices & $M$ & $S D$ & $M$ & $S D$ \\
\hline Processing Speed & $13.15^{\mathrm{b}}$ & 1.38 & $11.85^{\mathrm{b}}$ & 1.97 \\
\hline Working Memory & $6.27^{\mathrm{c}}$ & 0.62 & $4.84^{\mathrm{c}}$ & 0.82 \\
\hline Eyes Task Proportion & $6.23^{\mathrm{a}}$ & 1.77 & $5.45^{\mathrm{a}}$ & 1.60 \\
\hline Emotion Morph Proportion Correct & $0.744^{\mathrm{b}}$ & 0.090 & $0.673^{\mathrm{b}}$ & 0.129 \\
\hline Emotion Morph Reaction Time & $0.671^{\mathrm{c}}$ & 0.192 & $0.493^{\mathrm{c}}$ & 0.198 \\
\hline Emotion Morph Balanced Integration Score & $4.58^{\mathrm{c}}$ & 0.51 & $5.28^{\mathrm{c}}$ & 0.45 \\
\hline Emotion Photos Proportion & $0.948^{\mathrm{c}}$ & 0.808 & $-1.114^{\mathrm{c}}$ & 1.270 \\
\hline
\end{tabular}

Note. For each task, we compared young and older adults' performance using $t$-tests, with young adults always having better performance. Superscripts indicate the level of significant difference: ${ }^{a} p<$ $.05,{ }^{\mathrm{b}} p<.01,{ }^{\mathrm{c}} p<.001$.

Table 2 includes the correlations between variables. To provide maximum clarity, we used three measures for the Emotion Morph task: the Balanced Integration Score, proportion correct, and reaction time. The Balanced Integration Score is the most comprehensive measure and also tended to correlate most consistently with the other measures, so we retained this measure for the regressions below. Working 
memory and matrices correlated with all three tasks tapping social cognition, and processing speed correlated with one task (Emotion Morph). Advancing age correlated with worse performance on every task. The three tasks tapping social cognition correlated with each other.

\section{Table 2}

Correlations Between Main Variables in All Participants

$\begin{array}{lllllllll}1 & 2 & 3 & 4 & 5 & 6 & 7 & 8\end{array}$

\section{Age}

2. Matrices

$-.456^{\mathrm{C}}-$

3. Processing Speed $\quad-.745^{\mathrm{c}} \quad .448^{\mathrm{c}} \quad-$

\begin{tabular}{lllllllllll}
\hline 4. Working Memory & $-.287^{\mathrm{b}}$ & $.454^{\mathrm{c}}$ & $.305^{\mathrm{b}}$ & - & & & & \\
\hline 5. Emotion Photos & $-.281^{\mathrm{b}}$ & $.338^{\mathrm{b}}$ & .096 & $.292^{\mathrm{b}}$ & - & & & \\
\hline 6. Emotion Morphs BIS & $-.748^{\mathrm{c}}$ & $.384^{\mathrm{c}}$ & $.572^{\mathrm{c}}$ & $.317^{\mathrm{b}}$ & $.516^{\mathrm{c}}$ & - & & \\
\hline 7. Emotion Morph Prop & $-.435^{\mathrm{c}}$ & .167 & $.213^{\mathrm{a}}$ & .174 & $.607^{\mathrm{c}}$ & $.683^{\mathrm{c}}$ & - & \\
\hline 8. Emotion Morph RT & $.615^{\mathrm{c}}$ & $-.330^{\mathrm{b}}$ & -.599 & $-.233^{\mathrm{a}}$ & -.102 & $-.733^{\mathrm{c}}$ & -.041 & - \\
\hline 9. Eyes Task & $-.326^{\mathrm{b}}$ & $.309^{\mathrm{b}}$ & .117 & $.324^{\mathrm{b}}$ & $.432^{\mathrm{c}}$ & $.459^{\mathrm{c}}$ & $.340^{\mathrm{b}}$ & $-.271^{\mathrm{a}}$
\end{tabular}

Note. Emotion Morph BIS: Balanced Integration Score. Emotion Morph Prop: Proportion correct. Emotion Morph RT: reaction time. ${ }^{\mathrm{a}} p<.05,{ }^{\mathrm{b}} p<.01,{ }^{\mathrm{c}} p<.001$. (All tests two-tailed.)

We then used regression to examine whether the age group difference on each of the tasks tapping social cognition was still significant after controlling for general cognition. First, we examined the Emotion Morph BIS as the dependent variable, and included two steps in the regression, entering Age Group in the first step, and then the three general cognition tasks in the second step. Even though not all general cognition tasks were significant correlates of the tasks tapping social cognition, we entered all three in the second step to comprehensively control for cognition. Table 3 includes the results of this analysis for the Emotion Morph task. Recall that we posited that the Emotion Morph task should place the heaviest demands on general cognition because of the need for quick processing, yet only Age Group predicted unique variance.

When Emotion Photos was the dependent variable (Table 4), Age Group, Matrices and Processing Speed were all significant predictors. When the Eyes task was the dependent variable (Table 5), Age Group, Speed and Working Memory were all significant predictors. To summarize, for all three tasks measuring social cognition, Age Group continued to predict social cognition after accounting for general cognition, 
with processing speed predicting unique variance twice, and working memory and matrices predicting unique variance once each.

\section{Table 3}

Regression Predicting Emotion Morph Balanced Integration Score (Speed + Accuracy)

$\begin{array}{lllll}B & \text { Beta } & t & p & D R^{2}\end{array}$

\section{Step 1} .497

Age Group

$-2.062$

$-.705$

$-9.17$

$<.001$

Step 2

.035

$\begin{array}{lcccc}\text { Age Group } & -1.735 & -.593 & -5.52 & <.001 \\ \text { Matrices } & .063 & .076 & 0.84 & .405 \\ \text { Processing Speed } & .114 & .079 & 0.70 & .486 \\ \text { Working Memory } & .104 & .123 & 1.44 & .153\end{array}$

Note. $D R^{2}$ represents the additional variance accounted for by all the variables in a step. Regression statistics given with relevant variables at a step in the prediction equation (i.e., Age Group at Step 1, and all four variables at Step 2).

\section{Table 4}

Regression Predicting Emotion Photos Performance
$B$
Beta
$t$
$p$
$D R^{2}$

Step 1

.061

Age Group

$-.050$

$-.247$

$-2.35$

.021

\section{Step 2}

\begin{tabular}{lllll} 
Age Group & -.068 & -.332 & -2.36 & .021 \\
\hline Matrices & .016 & .275 & 2.30 & .024 \\
\hline Processing Speed & -.032 & -.321 & -2.18 & .032 \\
Working Memory & .011 & .190 & 1.70 & .093
\end{tabular}

Note. $D R^{2}$ represents the additional variance counted for by all the variables in a step. Regression statistics given with relevant variables at a step in the prediction equation. 
Regression Predicting Eyes Task Performance
$B$
Beta
$t$
$p$
$D R^{2}$

\section{Step 1}

.097
Age Group
$-.2 .56$
$-.312$
$-3.02$
.003

Step 2

.138

$\begin{array}{lllll}\text { Age Group } & -3.60 & -.438 & -3.18 & .002 \\ \text { Matrices } & 0.46 & .199 & 1.70 & .092 \\ \text { Processing Speed } & -1.45 & -.357 & -2.48 & .015 \\ \text { Working Memory } & 0.58 & .243 & 2.22 & .029\end{array}$

Note. $D R^{2}$ represents the additional variance counted for by all the variables in a step. Regression statistics given with relevant variables at a step in the prediction equation.

\section{Discussion}

Older adults typically do worse on tests of both general and social cognition, leading to the suggestion that worse general cognition underlies worse social cognition. Yet, previous research is equivocal. General cognition sometimes correlates with social cognition and sometimes age differences are eliminated after accounting for general cognition. However, sometimes general cognition doesn't correlate and age differences in social cognition persist.

One of the difficulties in interpreting previous findings is that the studies have usually examined only one of the three core cognitive abilities (processing speed, working memory, and fluid IQ) at a time. Indeed, 9 of 14 studies that have provided information on these questions have included a single task to measure general cognition, and 9 of 14 have, likewise, provided a single task to measure emotion recognition. It seems likely that better coverage of both general cognition and social cognition in an individual study would provide more comprehensive information as to their relation. Further, it seems important to choose tasks with varying cognitive demands; if these are central to performance, then relations with more cognitively demanding tasks should ensue. Thus, we examined this issue using a battery of tasks tapping both general cognition and social cognition.

Further, the three tasks measuring social cognition had varying cognitive demands. The Eyes task (which presented just the eyes and taps an understanding of complex emotions and mental states) used still photos. The Emotion Photos task (a classic test of ER) also used still photos, but examined just basic emotions. Neither the Eyes nor the Emotion Photos task had a time pressure or a need to integrate information over time. However, the Emotion Morphs task had both of these requirements. Following 
previous researchers ${ }^{34}$, we posited that the cognitive demands were greater for the Emotion Morph task given these considerations because it required quick processing and updating of information.

As anticipated, older adults did worse than young adults on all three measures of general cognition, and the same was true for social cognition. Further, the three measures of general cognition often correlated with social cognition. Working memory and matrices correlated with all three tasks tapping social cognition, and processing speed correlated with the Emotion Morph task. However, in the regressions, whereas age group was a unique predictor of performance on all three tasks tapping social cognition, general cognition was less consistently a unique predictor. For instance, consider the Emotion Morph task, which (a) was dynamic, thereby placing greater demands on speed, working memory and fluid intelligence, even when considering just the proportion correct component ${ }^{34}$, (b) but also had a speeded component that would further accentuate the cognitive demands, and (c) older adults were substantially worse than young adults with the correlation between the BIS and age equal to -.748 (meaning there was ample age variance to explain). Nevertheless, none of the three general cognition measures predicted unique variance on the Emotion Morph task. In other words, although all three cognitive tasks correlated with the Emotion Morph BIS, and there was a large age effect to explain, it could not be explained by a decline in general cognition. In contrast, and counter-intuitively, two of the three cognitive measures did predict unique variance on the Emotion Photos and Eyes tasks. This pattern of correlations suggests a somewhat random relation between general cognition and social cognition.

Overall, the findings can be summed up as follows: (a) in agreement with some past studies, general cognition sometimes correlates with social cognition and sometimes explains unique variance, but (b) despite this, there was an age effect in every single instance, such that older adults had worse social cognition on all three tasks after controlling for general cognition. Because previous studies had obtained somewhat inconsistent results, but had used few tasks to index general and social cognition, there was an ongoing need for further study to clarify findings.

A strength of the present study was that we examined both general cognition and social cognition more extensively than in prior research with no prior study giving participants such a large number and range of tasks to measure each. More specifically, we measured the three core components of general cognition - speed, working memory and fluid intelligence - known to deteriorate over age. Another strength is that we purposely varied the cognitive demands of the social cognition measures. Thus, our study provides a more thorough test of these relations. Although we didn't measure some executive functions such as inhibitory ability, set shifting, or self-control, most social cognition measures (such as emotion recognition or the Eyes task) do not tax these skills to any obvious extent either. With such a large array of cognitive tasks possible, as well as tasks tapping social cognition (e.g., Strange Stories), no one study can do everything. Instead, we focussed on the most obvious cognitive correlates of the social cognitive tasks of interest.

At the outset, we outlined a number of theories as to why older adults have worse social cognition than young adults. The two theories that were difficult to rule out were general cognitive decline and brain 
decline. In fact, these two theories can be related at a deeper level because brain decline ultimately explains general cognitive decline. Thus, in our view, the most likely explanation is that some of the brain areas that mediate general cognition also mediate social cognition, yet with some distinction between these brain areas as well. For instance, working memory and fluid intelligence are thought to be primarily mediated by the dorsolateral prefrontal cortex ${ }^{43}$ as well as generalized changes in white matter ${ }^{44}$. Speed of processing is thought to be due to generalized changes in grey and white matter, including in frontal areas $^{45}$.

As for social cognition, there is some overlap in that there is also involvement of the dorsolateral prefrontal cortex ${ }^{46}$, yet there is also independence, with evidence that ER relies on regions such as the orbitfrontal cortex, the anterior cingulate cortex and temporal lobes ${ }^{2,4}$. Similarly, the Eyes task engages frontal and temporal brain regions 47,48 . There will be some degree of overlap in the rate of decline in all brain areas, depending on factors such as diet and exercise ${ }^{49}$. However, there is also evidence that these regions also undergo somewhat different rates of decline ${ }^{50,51}$. Thus, an analysis of brain regions and brain decline predicts both the overlap we obtained (i.e., the correlations between general cognition and social cognition), and the independence of these two abilities that we obtained.

In sum, we found that although aspects of general cognition correlate with success on tasks tapping social cognition, they do not fully explain age-related difficulties. Older adults continued to have difficulty on social cognitive tasks even after controlling for three core cognitive abilities known to decline over age.

\section{Method}

\section{Participants}

There were 47 young adults ( 9 males, 37 females, and 1 non-binary) ranging from 18 to 29 years old ( $\mathrm{M}=$ 19.62 years, $S D=1.68$ ) and 40 older adults (11 males and 29 females) ranging from 54 to 92 years old $(M=67.20$ years, $S D=8.67)$. The young adults were university students who participated as part of their psychology courses, and the older adults were recruited through a university database and paid a nominal fee for travel expenses.

The undergraduates were all at a similar stage of their education. For older adults, we coded education on a 1 to 6 scale (1: primary school, 2: some high school, 3: high school diploma, 4: community college or polytechnic, 5 : university undergraduate, 6 : university post-graduate). Older adults' education ranged from 1 to 6 with a mean of 2.59. To examine older adults' cognitive health we gave them the Mini-Mental State Examination ${ }^{52}$. The maximum score a participant could receive was 30 , with a score of 24 considered acceptable for a group with varying educational attainment. The mean score was 27.88 , with a range of 24 to 30 .

Participants received either course credit or travel money for their participation. Informed consent was provided by all participants. We carried out a power analysis using $\mathrm{G}^{\star} \mathrm{Power}^{53}$ to determine the power for 
detecting age group differences using a $t$-test, as well as significant correlations, with the following constraints: two tails (allowing for either young or older adults to have better performance) and allowing for a moderate effect size $(d=.30)$. The required sample size was 82 . Thus, our sample of 87 should have been adequate to detect age group differences.

\section{Materials and Procedure}

\section{Matrices.}

The stimuli were pictures from the Wechsler Adult Intelligence Scale (WAIS-IV) ${ }^{54}$. The test is designed to measure visual processing and abstract spatial perception (i.e., fluid intelligence). Fourteen different matrices were used in the present experiment, which included two practice trials and 12 test trials. For each item, participants viewed an array of pictures with one missing square. Participants were told to select the picture that fitted the array from the five options presented below the picture, with the total score used in analyses..

\section{Processing Speed.}

We recorded the number of clicks a participant could make with a mouse in 25 seconds.

\section{Working Memory.}

We used a backwards digit span test ${ }^{54}$ to examine working memory. Participants were asked to verbally recall the numbers in the reverse order they heard them. There were eight trials, consisting of two items each of three digits, four digits, five digits and six digits. On each trial the numbers were spoken by the experimenter at a rate of one number per second and participants were allowed to hear the set of numbers twice before giving their answer. Correct performance required the participant to repeat all digits in the correct (reversed) order.

\section{Emotion Morph.}

The stimuli were black-and-white images selected from the Facial Expressions of Emotion Test ${ }^{55}$, which were manipulated to create six-second videos in which the face morphed from one emotion to another. In total there were 12 items, with two items (final emotion) for each of the six basic emotions. On each trial, the face started by expressing one emotion and morphed into one of the other five basic emotions. Participants were asked to stop the array when they could identify the new emotion, with the six basic emotions given on an answer sheet. The stimuli were created using MorphX ${ }^{56}$. In the descriptive 
statistics, we report proportion correct, reaction time, and a Balanced Integration Score ${ }^{42}$. In the subsequent analyses, we used the BIS given that both speed and accuracy are relevant to task success.

\section{Still Emotion Recognition.}

The stimuli for this task were still images selected from the Emotion Morph task. Participants were asked to identify the emotions of 30 facial expressions. This included one $100 \%$ version of the angry, sad, fearful, disgusted, surprised and happy faces used in the Emotion Morph task (six images in total), and four $65 \%$ versions of these faces (with $35 \%$ being another of the six basic emotions). We used $65 \%$ because that presented a combination that was more subtle than the $100 \%$ versions, but still clearly identifiable as a particular expression. The morphs were included to increase the difficulty level and make the task more sensitive to potential age differences in recognition. Images remained on the screen and participants had an unlimited time to choose from amongst six emotion labels (angry, sad, etc.), with total proportion correct used in the analyses.

\section{Eyes Task.}

We gave participants the 36 items of the revised version of the Adult "Reading the Mind in the Eyes" test ${ }^{57}$. Each item included a still black-and-white photograph of the eye region of the face, along with four corresponding words presented on the corners of the screen. Images remained on the screen and participants had an unlimited time to choose the word that best described the person's feelings or thoughts, with total proportion correct used in the analyses.

\section{Ethics.}

This study was approved by the University of Otago Human Ethics Committee (reference number 19/140) and carried out consistent with American Psychological Association ethical guidelines. Prior to beginning, informed consent was obtained from all participants.

\section{Declarations}

\section{Author Contributions}

Nicholas Currie collected the data. Kangning Du oversaw the preparation of tasks and collection of data. Qiuyi Kong oversaw the preparation of tasks and collection of data, and helped to write a draft of the article. Ted Ruffman oversaw the planning of the study, analyses and helped to write a draft of the article.

\section{Additional Information}


None of the authors have competing financial or non-financial interests.

\section{References}

1. Hayes, G. S., McLennan, S. N., Henry, J. D., Phillips, L. H., Terrett, G., Rendell, P. G., Pelly, R. M., \& Labuschagne, I. (2020). Task characteristics influence facial emotion recognition age-effects: A meta-analytic review. Psychology and Aging, 35(2), 295-315. DOI: 10.1037/pag0000441

2. Ruffman, T., Henry, J. D., Livingstone, V., \& Phillips, L. H. (2008). A meta-analytic review of emotion recognition and aging: Implications for neuropsychological models of aging. Neuroscience \& Biobehavioral Reviews, 32(4), 863-881. DOI: 0.1016/j.neubiorev.2008.01.001

3. Henry, J. D., Phillips, L. H., Ruffman, T., \& Bailey, P. E. (2013). A meta-analytic review of age differences in theory of mind. Psychology and Aging, 28(3), 826. DOI: 10.1037/a0030677

4. Ruffman, T., \& Sutcliffe, R. (2020). Emotion recognition and aging of the social brain. In A. K. Thomas, \& A. Gutchess (Eds.), Handbook of cognitive aging, Part III - Aging in a socioemotional context (pp.367-382).

5. Fry, A. F., \& Hale, S. (1996). Processing speed, working memory, and fluid intelligence: Evidence for a developmental cascade. Psychological Science, 7(4), 237-241. DOI: 10.1111/j.14679280.1996.tb00366.x

6. Salthouse, T. A. (1996). The processing-speed theory of adult age differences in cognition. Psychological Review, 103(3), 403-428.

7. Salthouse, T. A. (2010). Selective review of cognitive aging. Journal of the International Neuropsychological Society, 16(5), 754-760. DOI: 10.1017/S1355617710000706

8. Salthouse, T. A., \& Pink, J. E. (2013). Why is working memory related to fluid intelligence? Psychonomic Bulletin and Review, 15(2), 364-371. DOI: 10.3758/PBR.15.2.364

9. Hildebrandt, A., Sommer, W., Schacht, A., \& Wilhelm, O. (2015). Perceiving and remembering emotional facial expressions - A basic facet of emotional intelligence. Intelligence, 50, 52-67. DOI: 10.1016/j.intell.2015.02.003

10. Lewis, G. J., Lefevre, C. E., \& Young, A. W. (2016). Functional architecture of visual emotion recognition ability: A latent variable approach. Journal of Experimental Psychology: General, 145(5), 589-602. DOI: $10.1037 / x g e 0000160$

11. Schlegel, K., Palese, T., Schmid Mast, M., Rammsayer, T. H., Hall, J. A., \& Murphy, N. A. (2020). A metaanalysis of the relationship between emotion recognition ability and intelligence, Cognition and Emotion, 34(2), 329-351. DOI: 10.1080/02699931.2019.1632801

12. Wang, J.-J., \& Kaufman, A. S. (1993). Changes in fluid and crystallized intelligence across the 20 - to 90-year age range on the K-Bit. Journal of Psychoeducational Assessment, 11(1), 29-37. DOI: 10.1177/073428299301100104

13. Connolly, H. L., Young, A. W., \& Lewis, G. J. (2021). Face perception across the adult lifespan: Evidence for age-related changes independent of general intelligence. Cognition and Emotion, 35(5), 
890-901. DOI: 10.1080/02699931.2021.1901657

14. Horning, S. M., Cornwell, R. E., \& Davis, H. P. (2012). The recognition of facial expressions: An investigation of the influence of age and cognition. Aging, Neuropsychology, and Cognition, 19(6), 657-676. DOI: 10.1080/ 138255820.2011.645011

15. Murphy, J., Millgate, E., Geary, H., Catmur, C., \& Bird, G. (2019). No effect of age on emotion recognition after accounting for cognitive factors and depression. Quarterly Journal of Experimental Psycholoogy, 72(11), 2690-2704. DOI: 10.1177/175702181985914

16. Murphy, N. A., \& Isaacowitz, D. M. (2010). Age effects and gaze patterns in recognising expressions: An in-depth look at gaze measures and covariates. Cognition and Emotion, 24(3), 436-452. DOI: $10.1080 / 02699930802664623$

17. Orbelo, D. M., Grim, M. A., Talbott, R. E., \& Ross, E. D. (2005). Impaired comprehension of affective prosody in elderly subjects is not predicted by age-related hearing loss or age-related cognitive decline. Journal of Geriatric Psychiatry and Neurology, 18(1), 25-32. DOI: $10.1177 / 0891988704272214$

18. Orgeta, V., \& Phillips, L. H. (2007). Effects of age and emotional intensity on the recognition of facial emotion. Experimental Aging Research, 34(1), 63-79. DOI: 10.1080/03610730701762047

19. Ruffman, T., Sullivan, S., \& Dittrich, W. (2009). Older adults' recognition of bodily and auditory expressions of emotion. Psychology and Aging, 24(3), 614-622. DOI: 10.1037/a0016356

20. Ryan, M., Murray, J., \& Ruffman, T. (2010). Aging and the perception of emotion: Processing vocal expressions alone and with faces. Experimental Aging Research, 36(1), 1-22. DOI:

$10.1080 / 03610730903418372$

21. Sutcliffe, R., Rendell, P. G., Henry, J. D., Bailey, P. E., \& Ruffman, T. (2017). Music to my ears: Agerelated decline in musical and facial emotion recognition. Psychology and Aging, 32(8), 698-709. DOI: $10.1037 /$ pag0000203

22. Zhao, M.-F., Zimmer, H. D., \& Shen, X. (2016). Exploring the cognitive processes causing the agerelated categorization deficit in the recognition of facial expressions. Experimental Aging Research, 42(4), 348-364. DOI: 10.1080/0361073X.2016.1191854

23. Ebner, N. C., \& Johnson, M. K. (2009). Young and older emotional faces: Are there age-group differences in expression identification and memory? Emotion, 9(3), 329-339. DOI: $10.1037 / \mathrm{a} 0015179$

24. Keightley, M. L., Winocur, G., Burianova, H., Hongwanishkul, D., \& Grady, C. L. (2006). Age effects on social cognition: Faces tell a different story. Psychology and Aging, 20(3), 558-572. DOI: 10.1037/0882-7974.21.3.558

25. Sullivan, S., \& Ruffman, T. (2004a). Social cognition: How does it fare with advancing years? British Journal of Psychology, 95(1), 1-18. DOI: 10.1348/00071260432277942

26. Lambrecht, L., Kreifelts, B., \& Wildgruber, D. (2012). Age-related decrease in recognition of emotional facial and prosodic expressions. Emotion, 12(3), 529-539. DOI: 10.1037/ a0026827 
27. Lima, C. F., Alves, T., Scott, S. K., \& Castro, S. L. (2014). In the ear of the beholder: How age shapes emotion processing in nonverbal vocalizations. Emotion, 141(1), 145-160. DOI:

10.1080/02699931.2010.502449

28. Sullivan, S., \& Ruffman, T. (2004b). Emotion recognition deficits in the elderly. International Journal of Neuroscience, 114(3), 403-432. DOI: 10.1080/ 00207450490270901

29. Suzuki, A., \& Akiyama, H. (2013). Cognitive aging explains age-related differences in face-based recognition of basic emotions except for anger and disgust. Aging, Neuropsychology, and Cognition, 20(3), 253-270. DOI: 10.1080/13825585.2012.692761

30. Smith, M. L., Grühn, D., Bevitt, A., Ellis, M., Ciripan, O., Scrimgeour, S., Papasavva, M. \& Ewing, L. (2018). Transmitting and decoding facial expressions of emotion during healthy aging: More similarities than differences. Journal of Vision, 18(9): 10, 1-6. DOI: 10.1167/18.9.10

31. West, J. T., Horning, S. M., Klebe, K. J., Foster, S. M., Cornwell, E., Perrett, D., Burt, D. M., \& Davis, H. P. (2012). Age effects on emotion recognition in facial displays: From 20 to 89 years of age. Experimental Aging Research, 38(2), 146-168. DOI: 10.1080/0361073X.2012.659997

32. Duncan, J., Chylinski, D., Mitchell, D. J., \& Bhandari, A. (2017). Complexity and compositionality in fluid intelligence. Proceedings of the National Academy of Science, 114(20), 5295-5299. DOI: $10.1073 /$ pnas. 1621147114

33. Isaacowitz, D. M. \& Tehan Stanley, J. T. (2011). Bringing an ecological perspective to the study of aging and recognition of emotional facial expressions: Past, current, and future methods. Journal of Nonverbal Behavior, 35(4), 261-278. DOI: 10.1007/s10919-011-0113-6

34. Spreij, L. A., Ten Brink A. F., Visser-Meily, J. M. A., \& Nijboer, T. C. W. (2020). Increasing cognitive demand in assessments of visuo-spatial neglect: Testing the concepts of static and dynamic tests. Journal of Clinical and Experimental Neuropsychology, 42(7), 675-689. DOI: 10.1080/13803395.2020.1798881

35. Maylor, E. A., Moulson, J. M., Muncer, A.-M., \& Taylor, L. A. (2002). Does performance on theory of mind tasks decline in old age? British Journal of Psychology, 93(4), 465-485. DOI: $10.1348 / 000712602761381358$

36. Moran, J. M. (2013). Lifespan development: The effects of typical aging on theory of mind. Behavioural Brain Research, 237, 32-40. DOI: 10.1016/j.bbr.2012.09.020

37. Johansson Nolaker, E., Murray, K., Happé, F., \& Charlton, R. A. (2018). Cognitive and affective associations with an ecologically valid test of theory of mind across the lifespan. Neuropsychology, 32(6), 754-763. DOI: 10.1037/neu0000464

38. Cho, I., \& Cohen, A. S. (2019). Explaining age-related decline in theory of mind: Evidence for intact competence but compromised executive function. PLOSONE,14(9): e0222890. DOI:

10.1371 /journal.pone.0222890

39. Di Tella, M., Ardito, R. B., Dutto, F., \& Adenzato, M. (2020). On the (lack of) association between theory of mind and executive functions: A study in a non-clinical adult sample. Scientific Reports, 10: 17283. DOI: 19.1038/s41598-020-74476-0 
40. Otsuka, Y., Shizawa, M., Sato, A., \& Itakura, S. (2021). The role of executive functions in older adults' affective theory of mind. Archives of Gerontology and Geriatrics, 97, 104513. DOI:

10.1016/j.archger.2021.104513

41. Oakley, B. F. M., Brewer, R., Bird, G., \& Catmure, C. (2016). Theory of mind is not theory of emotion: A cautionary note on the Reading the Mind in the Eyes Test. Journal of Abnormal Psychology, 125(6), 818-823. DOI: 10.1037/abn0000182

42. Liesefeld, H., \& Janczyk, M. (2019). Combining speed and accuracy to control for speed-accuracy trade-offs(?). Behavior Research Methods, 51(1), p.40-60. DOI: 10.3758/s13428-018-1076-x

43. Levy, R., \& Goldman-Rakic, P. S. (2000). Segregation of working memory functions within the dorsolateral prefrontal cortex. Experimental Brain Research, 133(1), 23-32. DOI: 10.1007/ s002210000397

44. Deary, I. J., Leaper, S. A., Murray, A. D., Staff, R. T., \& Whalley, L. J. (2003). Cerebral white matter abnormalities and lifetime cognitive change: A 67-year follow-up of the Scottish Mental Survey of 1932. Psychology and Aging, 18(1), 140-148. DOI: 10.1037/0882-7974.18.1.140

45. Eckert, M. A., Keren, N. I., Roberts, D. R., Calhoun, V. D., \& Harris, K. C. (2010). Age-related changes in processing speed: Unique contributions of cerebellar and prefrontal cortex. Frontiers in Human Neuroscience, 4: 10. DOI: 10.3389/neuro.09.010.2010

46. Nejati, V., Majdi, R., Salehinejad, M. A., \& Nitsche, M. A. (2021). The role of dorsolateral and ventromedial prefrontal cortex in the processing of emotional dimensions. Scientific Reports, 11: 1971. DOI: $10.1038 / \mathrm{s} 41598-021-81454-7$

47. Sato, W., Kochiyama, T., Uono, S., Sawada, R., Kubota, Y., Yoshimura, S., \& Toichi, M. (2016). Structural neural substrates of reading the mind in the eyes. Frontiers in Human Neuroscience, 10 : 151. DOI: 10.3389/fnhum.2016.00151

48. Thye, M. D., Murdaugh, D. L., \& Kana, R. K. (2018). Brain mechanisms underlying reading the mind from eyes, voice, and actions. Neuroscience, 374, 172-186. DOI: 10.1016/j.neuroscience.2018.01.045

49. Baranowski, B. J., Marko, D. M., Fenech, R. K., Yang, A. J. T., \& MacPherson, R. E. K. (2020). Healthy brain, healthy life: A review of diet and exercise interventions to promote brain health and reduce Alzheimer's disease risk. Applied Physiology, Nutrition and Metabolism, 45(10), 1055-1065. DOI: 10.1139/apnm-2019-0910

50. Iveson, M. H., Della Sala, S., \& MacPherson, S. E. (2020). Does age affect medial prefrontal functions? A behavioral investigation. Psychology and Neuroscience, 13(3), 390-405. DOI: 10.1037/pne0000194

51. Rajah, M. N., \& D’Esposito, M. (2005). Region-specific changes in prefrontal function with age: A review of PET and fMRI studies on working and episodic memory. Brain, 128(9), DOI:

10.1093/brain/awh608

52. Folstein, M. F., Folstein, S. E., \& McHugh, P. R. (1975). "Mini-mental state”: A practical method for grading the cognitive state of patients for the clinician. Journal of Psychiatric Research, 12(3), 189198. DOI: 10.1016/0022-3956(75)90026-6

Page 18/19 
53. Faul, F., Erdfelder, E., Buchner, A., \& Lang, A.-G. (2009). Statistical power analyses using G*Power 3.1: Tests for correlation and regression analyses. Behavior Research Methods, 41(4), 1149-1160. DOI: 10.3758/BRM.41.4.1149

54. Wechsler, D. (2008). Wechsler Adult Intelligence Scale - Fourth Edition (WAIS-IV). DOI: 10.1037/t15169-000

55. Young, A., Perrett, D. I., Calder, A., Sprengelmeyer, R. H., \& Ekman, P. (2002). Facial expressions of emotion: Stimuli and Test (FEEST). Oxford, UK: Pearson.

56. Wenneberg, M. (2015). MorphX. 2.7.1 https://apps.apple.com/us/app/morphx/id413655396?mt=12 Accessed 04.03.19.

57. Baron-Cohen, S., Wheelwright, S., Hill, J., Raste, Y., \& Plumb, I. (2001). The "Reading the Mind in the Eyes" test revised version: A study with normal adults, and adults with Asperger syndrome or highfunctioning autism. Journal of Child Psychology and Psychiatry, 42(2), 241-251. DOI: $10.1017 /$ S0021963001006643 